\title{
RELXILL_NK: A Relativistic Reflection Model for Testing Einstein's Gravity
}

\author{
Cosimo Bambi ${ }^{1,2, *}$, Askar B. Abdikamalov ${ }^{1}$, Dimitry Ayzenberg ${ }^{1}$, Zheng Cao ${ }^{1}$, Honghui Liu ${ }^{1}(\mathbb{D}$, \\ Sourabh Nampalliwar ${ }^{2} \odot$, Ashutosh Tripathi ${ }^{1}$, Jingyi Wang-Ji ${ }^{1,3}$ and Yerong $\mathrm{Xu}^{1}$ \\ 1 Center for Field Theory and Particle Physics and Department of Physics, Fudan University, \\ Shanghai 200438, China; 17110190057@fudan.edu.cn (A.B.A.); dimitry@fudan.edu.cn (D.A.); \\ 13307110005@fudan.edu.cn (Z.C.); 15307110164@fudan.edu.cn (H.L.); 16110190056@fudan.edu.cn (A.T.); \\ 14307110368@fudan.edu.cn (J.W.-J.); 15307110047@fudan.edu.cn (Y.X.) \\ 2 Theoretical Astrophysics, Eberhard-Karls Universität Tübingen, 72076 Tübingen, Germany; \\ sourabhnampalliwar@gmail.com \\ 3 MIT Kavli Institute for Astrophysics and Space Research, MIT, Cambridge, MA 02139, USA \\ * Correspondence: bambi@fudan.edu.cn
}

Received: 7 June 2018; Accepted: 9 July 2018; Published: 11 July 2018

\begin{abstract}
Einstein's theory of general relativity was proposed over 100 years ago and has successfully passed a large number of observational tests in the weak field regime. However, the strong field regime is largely unexplored, and there are many modified and alternative theories that have the same predictions as Einstein's gravity for weak fields and present deviations when gravity becomes strong. RELXILL_NK is the first relativistic reflection model for probing the spacetime metric in the vicinity of astrophysical black holes and testing Einstein's gravity in the strong field regime. Here, we present our current constraints on possible deviations from Einstein's gravity obtained from the black holes in 1H0707-495, Ark 564, GX 339-4, and GS 1354-645.
\end{abstract}

Keywords: general relativity; black boles; X-ray astronomy

\section{Introduction}

Einstein's theory of gravity, the theory of general relativity, has undergone a variety of observational tests since it was first proposed a century ago, primarily with experiments in the Solar System and radio observations of binary pulsars [1]. While it has largely been successful, questions remain on its validity in the so-called strong field, or ultraviolet (UV), regime ${ }^{1}$. There are indeed many modified and alternative theories that have the same predictions as Einstein's gravity for weak fields and present deviations when gravity becomes strong. Testing strong gravity is among the priorities in contemporary physics.

Astrophysical black holes are the most extreme objects that can be found in the Universe and ideal laboratories for testing strong gravity [2-6]. In four-dimensional Einstein's gravity, the only stationary and asymptotically flat, vacuum black hole solution, which is regular on and outside of the event horizon, is the Kerr metric [7]. Kerr black holes are relatively simple objects, and are completely described by only two parameters, the mass $M$ and the spin angular momentum $J$ of the black hole. This is the result of the celebrated "no-hair theorems" [8-10]. Hairy black holes are possible, for instance, in the presence of exotic fields [11,12].

1 We note that questions remain on the validity of general relativity even at large scales and extremely weak gravitational fields, i.e. the infrared (IR) regime. Tests of general relativity in the IR regime are mainly motivated by the issues of dark matter and dark energy. 
It is remarkable that the spacetime metric around an astrophysical black hole formed from gravitational collapse should be well approximated by the stationary Kerr solution of Einstein's gravity. After the gravitational collapse of the progenitor body and the formation of the black hole, the spacetime quickly moves to the Kerr solution with the emission of gravitational waves $[13,14]$ ("black holes rapidly go bald"). Astrophysical bodies can have a non-vanishing electric charge. However, the equilibrium electric charge for a macroscopic black hole is very small and completely negligible for the spacetime metric $[15,16]$. The presence of an accretion disk around the black hole, or of a companion star orbiting the black hole, has a very small impact on the background metric in the strong gravity regions and can be safely ignored [17,18]. For example, the ratio between the mass of the accretion disk and the mass of the black hole in an X-ray binary system can be $m / M \sim 10^{-10}$. This is a small quantity that can be used as an expansion parameter to take into account the correction on the background metric and, even assuming that the mass of the accretion disk was at a point close to the black hole, the correction to the Kerr metric would be of the order of $m / M$. If we consider the companion star, the expansion parameter would be $M_{\mathrm{c}} / r<10^{-6}$, where $M_{\mathrm{c}}$ and $r$ are, respectively, the mass of the companion star and the distance from the companion star. Even in this case, we can expect corrections to the Kerr metric of the order of $M_{\mathrm{c}} / r$ and are negligible. In the end, macroscopic deviations for the Kerr solution may be possible in the presence of new physics, such as modified classical gravity [19], quantum gravity effects [20-22], or exotic matter [12,23].

From astrophysical observations, we know two main types of astrophysical black holes. Stellar-mass black holes $\left(M \approx 3-100 M_{\odot}\right)$ are the natural product of the gravitational collapse of heavy stars, after the latter have exhausted all their nuclear fuel [24]. Supermassive black holes $\left(M \sim 10^{5}-10^{10} M_{\odot}\right)$ are found at the center of many galaxies [25]. All these objects are thought to be black holes because this is the most natural interpretation in the framework of conventional physics. For example, in the case of stellar-mass black holes in X-ray binary systems, we can get a dynamical measurement of the mass of the black hole. If the latter exceeds $3 M_{\odot}$, which is the maximum mass for a neutron star [26,27], and we can exclude it is a normal star, the most natural explanation is that it is a black hole. In the case of the supermassive black hole at the center of the Galaxy, we can argue it is too massive, compact, and old to be a cluster of non-luminous bodies like neutron stars [28]. The non-detection of electromagnetic radiation from the putative surface of all these objects is also consistent with the conjecture that they are instead a black hole with an event horizon $[29,30]$. The recent detections of gravitational waves are consistent with the signals expected from black holes in general relativity [31,32], even if current data cannot put strong constraints on alternative scenarios [33,34]. Despite this body of evidence, we still do not know if the spacetime metric around these objects is described by the Kerr solution, as would be required in Einstein's gravity.

\section{X-Ray Reflection Spectroscopy}

The standard approach to analyze black holes in an astrophysical setting is the disk-corona model [16,35], where black holes are surrounded by a geometrically thin disk and possess a "corona", as shown in Figure 1. In the case of a stellar-mass black hole in a binary system, the material of the disk comes from the companion star. In the case of a supermassive black hole in a galactic nucleus, the disk is created by the material of the interstellar medium around the object. The disk emits like a blackbody locally, and as a multi-temperature blackbody when integrated radially (this is known as the thermal component). The temperature of the accretion disk depends on the black hole mass, the mass accretion rate, and the radial coordinate of the emission point in the accretion disk. For a black hole accreting at about $10 \%$ of its Eddington limit, the thermal spectrum of the inner part of the accretion disk is in the soft X-ray band (0.1-1 keV) for stellar-mass black holes and in the optical/UV band $(1-10 \mathrm{eV})$ for the supermassive ones. The corona is a hotter $(\sim 100 \mathrm{keV})$, usually optically thin, cloud near the black hole. For instance, it may be the base of a jet or some atmosphere above the accretion disk, but the exact morphology is not well understood. 
Thermal photons from the disk can gain energy via inverse Compton scattering off the hot electrons in the corona, and transform into X-rays with a characteristic power-law component. These reprocessed photons in turn illuminate the disk, producing a reflection component with fluorescent emission lines. The most prominent feature of the reflection spectrum is usually the iron $\mathrm{K} \alpha$ line, with emission lines at $6.4 \mathrm{keV}$ in the case of neutral or weakly ionized iron and shifts up to $6.97 \mathrm{keV}$ for H-like ions.

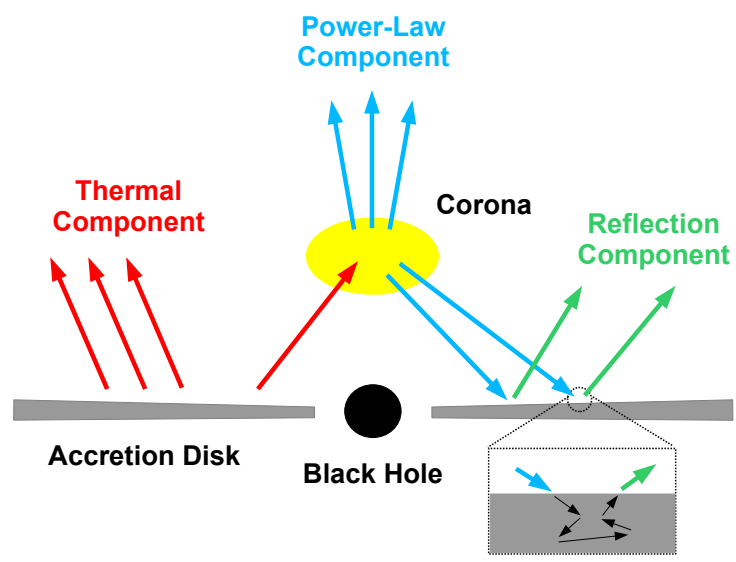

Figure 1. Disk-corona model.

While the iron $\mathrm{K} \alpha$ line is a narrow line in the rest-frame of the gas, relativistic effects due to the gravity of the central black hole cause this line to be broadened and skewed for observers far away. In the presence of high quality data and with the correct astrophysical model, analysis of the reflection spectrum can be a powerful tool for probing the strong gravitational fields of accreting black holes. The method was originally proposed and developed for measuring black hole spins under the assumption that the metric around astrophysical black holes is described by the Kerr solution [36,37]. More recently, the technique has been proposed for testing Einstein's gravity in the strong field regime [38-51]. Note that spin measurements (if we assume the Kerr metric) or tests of the Kerr metric require fitting the whole reflection spectrum, not just the iron line, even if often (but not always) the iron line is the feature that primarily determines the measurement of the parameters of the background metric in the strong gravity region.

\section{The Relativistic Reflection Model RELXILL_NK}

\subsection{RELXILL}

Currently, the most advanced model for the calculation of the reflection spectrum of the accretion disk around Kerr black holes is RELXILL [52,53]. RELXILL is the result of the merger of the reflection code XILLVER [54,55] with the relativistic convolution model RELCONV [56]. We can write

RELXILL $\sim$ RELCONV $\times$ XILLVER.

XILLVER provides the reflection model in the rest-frame of the emitting gas at every point of the accretion disk. Compared to all earlier codes, XILLVER has a superior treatment of the radiative transfer and of the ionization balance, by implementing the most complete atomic database for modeling synthetic photoionized X-ray spectra. The microphysics captured by XILLVER is much more rigorous than for any earlier code, principally because of the detailed treatment of the K-shell atomic properties of the prominent ions. Despite that, there are still a number of limitations in this reflection model: the electron density is fixed to $10^{15} \mathrm{~cm}^{-3}$ and independent of vertical height; with the exception of iron, 
all elemental abundances are assumed to be solar, thermal photons of the accretion disk are ignored in the radiative transfer calculations, etc.

RELCONV is a convolution model. It requires as input the reflection spectrum in the rest frame of the gas at every point of the accretion disk and provides as output the reflection spectrum of an accretion disk around a Kerr black hole as it would be detected by a faraway observer. The model assumes that the accretion disk is infinitesimally thin and lies on the equatorial plane, perpendicular to the black hole spin. The particles of the gas of the accretion disk are supposed to follow nearly geodesic circular orbits on the equatorial plane. With a similar set-up, RELCONV integrates the reflection spectrum of the disk over all radii and includes the relativistic effects in a Kerr spacetime affecting the photons propagating from the emission point on the disk to the detection point in the flat faraway region.

\subsection{Testing the Kerr Black Hole Hypothesis}

There are two natural approaches to test the spacetime metric around astrophysical black holes. They can be called, respectively, top-down and bottom-up methods.

In the top-down approach, we want to test a specific alternative theory of gravity in which uncharged black holes are not described by the Kerr solution. In such a case, we fit the astrophysical data both with a Kerr model and with a model employing the non-Kerr spacetime of the gravity theory under investigation. Depending on the quality of the available data and of the theoretical model, we may be able to check whether the astrophysical observations prefer the Kerr metric of Einstein's gravity or the non-Kerr solution of the alternative theory. There are two problems if we follow this method. Firstly, there are a large number of alternative scenarios to Einstein's gravity, and none seems to be more motivated than the others, so we should repeat this study for every alternative theory of gravity. Secondly, typically we do not know the rotating black hole solutions in alternative theories of gravity. The problem is in solving the corresponding field equations for a stationary and axisymmetric solution. Usually, we know the non-rotating solutions or some approximated solutions in the slow rotation limit, but only in exceptional cases do we know the complete rotating solutions.

In the bottom-up approach, we employ a parametrized metric to describe the background geometry of the spacetime in the astrophysical model. Such a parametrized metric is characterized by the mass $M$ and the spin angular momentum $J$ of the compact object generating the gravitational field, as well as by a number of "deformation parameters". If we set all the deformation parameters to zero, we have to recover the Kerr metric of Einstein's gravity. If at least one of the deformation parameters is non-vanishing, we have deviations from the Kerr metric. The strategy is thus to fit the astrophysical data with this parametrized model and try to measure the deformation parameters. If the astrophysical data require vanishing deformation parameters, the Kerr black hole hypothesis is verified. If at least one of the deformation parameters were non-vanishing, astrophysical data would prefer a spacetime metric different from the Kerr solution. In general, the parametrized metric and its deformation parameters do not have a clear physical meaning because the metric is not a solution of some alternative theory of gravity. Their significance instead lies in the fact that they capture deviations from a Kerr metric.

\subsection{RELXILL_NK}

Recently, we expanded the RELXILL model to RELXILL_NK, which incorporates non-Kerr metrics [57]. Since we only want to test the background metric around black holes, assuming that atomic physics is the same, the new model is

RELXILL_NK $\sim$ RELCONV_NK $\times$ XILLVER.

RELCONV_NK is a convolution model for non-Kerr metrics and we do not change the reflection code XILLVER. 
RELXILL_NK is presently the only reflection model for testing the strong gravity region of astrophysical black holes. In the current version, RELXILL_NK incorporates the non-Kerr metric proposed by Johannsen [58]. In Boyer-Lindquist coordinates, the line element of the Johannsen metric reads (we use units in which $G_{\mathrm{N}}=c=1$ ) [58]

$$
\begin{aligned}
d s^{2}= & -\frac{\tilde{\Sigma}\left(\Delta-a^{2} A_{2}^{2} \sin ^{2} \theta\right)}{B^{2}} d t^{2}-\frac{2 a\left[\left(r^{2}+a^{2}\right) A_{1} A_{2}-\Delta\right] \tilde{\Sigma} \sin ^{2} \theta}{B^{2}} d t d \phi \\
& +\frac{\tilde{\Sigma}}{\Delta A_{5}} d r^{2}+\tilde{\Sigma} d \theta^{2}+\frac{\left[\left(r^{2}+a^{2}\right)^{2} A_{1}^{2}-a^{2} \Delta \sin ^{2} \theta\right] \tilde{\Sigma} \sin ^{2} \theta}{B^{2}} d \phi^{2},
\end{aligned}
$$

where

$$
\begin{aligned}
& a=J / M, \quad B=\left(r^{2}+a^{2}\right) A_{1}-a^{2} A_{2} \sin ^{2} \theta, \quad \tilde{\Sigma}=\Sigma+f, \\
& \Sigma=r^{2}+a^{2} \cos ^{2} \theta, \quad \Delta=r^{2}-2 M r+a^{2},
\end{aligned}
$$

and the four free functions $f, A_{1}, A_{2}$, and $A_{5}$ are $^{2}$

$$
\begin{aligned}
& f=\sum_{n=3}^{\infty} \epsilon_{n} \frac{M^{n}}{r^{n-2}}, \\
& A_{1}=1+\sum_{n=3}^{\infty} \alpha_{1 n}\left(\frac{M}{r}\right)^{n}, \\
& A_{2}=1+\sum_{n=2}^{\infty} \alpha_{2 n}\left(\frac{M}{r}\right)^{n} \text {, } \\
& A_{5}=1+\sum_{n=2}^{\infty} \alpha_{5 n}\left(\frac{M}{r}\right)^{n} \text {. }
\end{aligned}
$$

The metric elements depend on the mass and spin of the black hole as well as on four free functions that measure potential deviations from the Kerr solution. The first order deformation parameters in these free functions are $\epsilon_{3}, \alpha_{13}, \alpha_{22}$, and $\alpha_{52}$. This metric exactly reduces to the Kerr solution for $\epsilon_{3}=\alpha_{13}=\alpha_{22}=\alpha_{52}=0$.

In the Kerr spacetime, the condition for the existence of an event horizon is $\left|a_{*}\right| \leq 1$, where $a_{*}=a / M=J / M^{2}$ is the dimensionless spin parameter. For $\left|a_{*}\right|>1$, there is no horizon, and the singularity at $r=0$ is naked. In the Johannsen spacetime, we still have the condition $\left|a_{*}\right| \leq 1$. Moreover, in order to exclude a violation of Lorentzian signature or the existence of closed time-like curves in the exterior region, we have to impose that the metric determinant is always negative and that $g_{\phi \phi}$ is never negative for radii larger than the radius of the event horizon. These conditions lead to the following constraints on the first-order deformation parameters [58]

$$
\begin{aligned}
& \alpha_{13}, \epsilon_{3} \geq-\left(1+\sqrt{1-a_{*}^{2}}\right)^{3}, \\
& \alpha_{22}, \alpha_{52} \geq-\left(1+\sqrt{1-a_{*}^{2}}\right)^{2} .
\end{aligned}
$$

2 The four free functions $f, A_{1}, A_{2}$, and $A_{5}$ are written as a power series in $M / r$

$$
f=\sum_{n=2}^{\infty} \epsilon_{n} \frac{M^{n}}{r^{n-2}}, \quad A_{1}=1+\sum_{n=0}^{\infty} \alpha_{1 n}\left(\frac{M}{r}\right)^{n}, \quad A_{2}=1+\sum_{n=0}^{\infty} \alpha_{2 n}\left(\frac{M}{r}\right)^{n}, \quad A_{5}=1+\sum_{n=0}^{\infty} \alpha_{5 n}\left(\frac{M}{r}\right)^{n} .
$$

In order to recover the correct asymptotic limit, we have to impose $\alpha_{10}=\alpha_{20}=\alpha_{50}=0$. We can set $\alpha_{11}=\alpha_{21}=\alpha_{51}=0$ without loss of generality because these parameters are related to the definition of $M$ and $a$ and do not have any direct physical implication. In order to satisfy Solar System experiments without fine-tuning, we require $\alpha_{12}=\epsilon_{2}=0$. Eventually, the first order deformation parameters without constraints from observations in weak gravitational fields are $\epsilon_{3}, \alpha_{13}, \alpha_{22}$, and $\alpha_{52}$. See Ref. [58] for more details. 
In the next section, we will review the constraints on $\alpha_{13}$ obtained so far from the analysis of X-ray data of some sources assuming that all the other deformation parameters vanish ${ }^{3}$ Since the metric is singular for $B=0$, we impose that $B$ never vanishes for radii larger than the event horizon, and this implies the following constraint on $\alpha_{13}$

$$
\alpha_{13} \geq-\frac{1}{2}\left(1+\sqrt{1-a_{*}^{2}}\right)^{4} .
$$

In the studies reported below, we only consider the parameter space satisfying the constraint in Equation (6), which is stronger than the requirement in Equation (5).

\section{Observational Constraints}

As of now, we have applied our RELXILL_NK to a few sources: the supermassive black holes in 1H0707-495 [59] and in Ark 564 [60], and the stellar-mass black holes in the X-ray binary systems GX 339-4 [61] and GS 1354-645 [62]. In what follows, we present the current constraints on the Johannsen deformation parameter $\alpha_{13}$ assuming that all the other deformation parameters vanish. Note that we have only analyzed a few sets of data of these objects. It is likely that a more detailed analysis of all the available X-ray data of these sources can provide stronger constraints on the deformation parameter $\alpha_{13}$.

\section{1. $1 H 0707-495$}

1H0707-495 is a Narrow Line Seyfert 1 galaxy. The supermassive black hole in its galactic nucleus looks to be a promising source for testing the Kerr metric using RELXILL_NK. The spectrum of this source shows significant edge features, which are commonly interpreted as an extremely strong reflection component. Moreover, previous studies that assumed the Kerr metric and a reflection dominated spectrum found that the inner radius of the accretion disk is very close to the compact object, and this increases the relativistic effect in the reflection spectrum.

In Ref. [59], we analyzed an observation with XMM-Newton of 2011 and, separately, three observations with NuSTAR (with simultaneous snapshots of Swift) of 2014. In the case of the 2011 data, we fit the data with two different models that provide equally good fits. In the first model, we employed the thermal disk model DISKBB to fit the "soft excess" around $1 \mathrm{keV}$, and the complete model is

TBABS $\times($ RELXILL_NK + DISKBB $)$.

In the second model, we employed a double reflection spectrum and the complete model is

TBABS $\times($ RELXILL_NK + RELXILL_NK $)$.

With the available data, it is not possible to say which one, if any, is correct or wrong because they both provide good fits. The resulting constraints on the spin parameter $a_{*}$ and the Johannsen parameter $\alpha_{13}$ are shown in Figure 2. The red, green, and blue lines indicate, respectively, the $68 \%$, $90 \%$, and $99 \%$ confidence level curves for two relevant parameters. The grayed region is ignored because those spacetimes violate the constraint in Equation (6). Note that the constraints shown in Figure 2 are slightly different from those reported in Figure 1 in Ref. [59] because those in Figure 2 have been obtained with a more recent version of RELXILL_NK and therefore they should be considered more reliable.

Our current version of RELXILL_NK is not ready to work with two or more free non-vanishing deformation parameters at the same time. Depending on the specific deformation parameters under consideration, there may be a degeneracy among them, which might be broken in the presence of high quality data or in combination with independent measurements. 


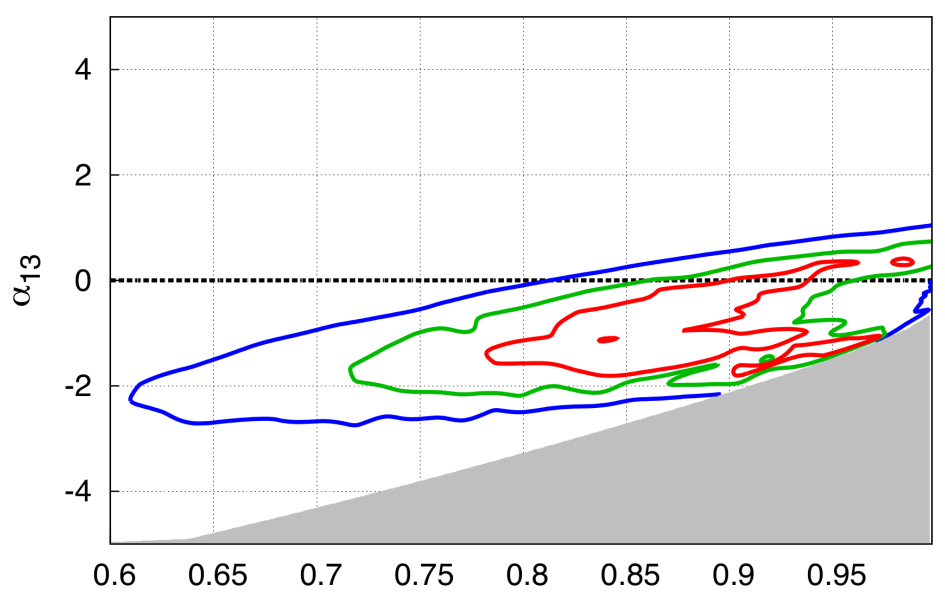

$a$ *

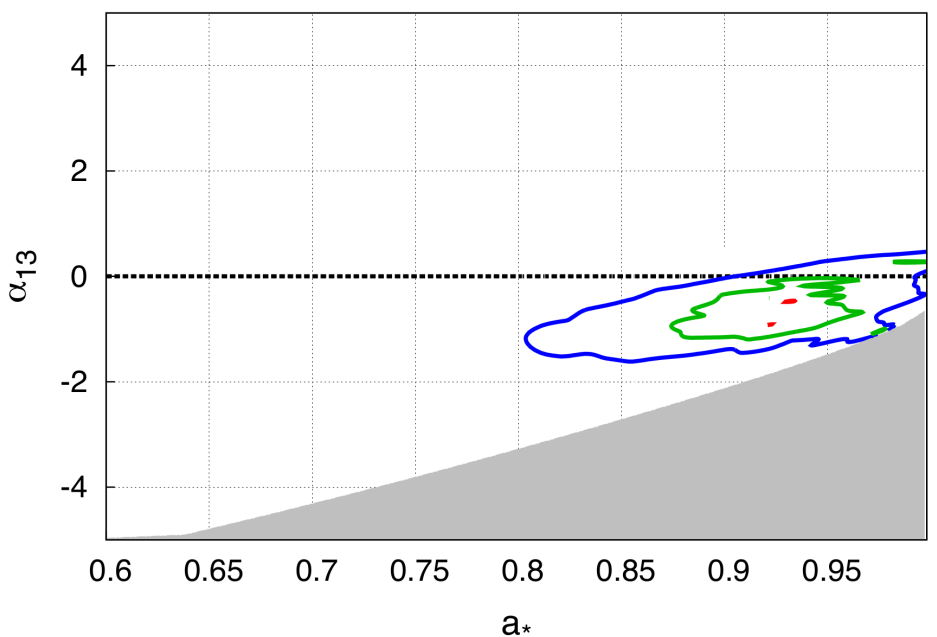

Figure 2. Constraints on the spin parameter $a_{*}$ and the Johannsen parameter $\alpha_{13}$ from the study in Ref. [59] of the 2011 XMM-Newton data of 1H0707-495 if we fit the "soft excess" around $1 \mathrm{keV}$ with a thermal model (top panel) or if we employ a double reflection model (bottom panel). The red, green, and blue lines indicate, respectively, the $68 \%, 90 \%$, and $99 \%$ confidence level curves for two relevant parameters. The grayed region is ignored because those spacetimes violate the constraint in Equation (6). See the text and Ref. [59] for more details. Note that the constraints reported in Ref. [59] are slightly different because that study used an earlier version of RELXILL_NK, and therefore the constraints reported here should be more reliable.

For the 2014 observations of NuSTAR and Swift, we can obtain a good fit already with the model

TBABS $\times$ RELXILL_NK.

The constraints on $a_{*}$ and $\alpha_{13}$ are shown in Figure 3. The red, green, and blue lines indicate, respectively, the $68 \%, 90 \%$, and $99 \%$ confidence level curves for two relevant parameters and the grayed region is ignored. Even in this case, the constraints in Figure 3 are slightly different from those reported in Figure 2 in Ref. [59] because they have been obtained with the latest version of RELXILL_NK and they are thus more reliable. 


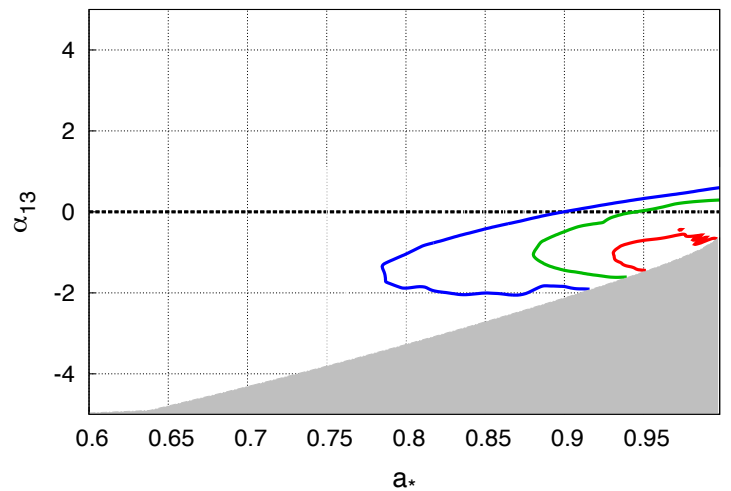

Figure 3. Constraints on the spin parameter $a_{*}$ and the Johannsen parameter $\alpha_{13}$ from the study in Ref. [59] of NuSTAR and Swift data of 1H0707-495. The red, green, and blue lines indicate, respectively, the $68 \%, 90 \%$, and $99 \%$ confidence level curves for two relevant parameters. The grayed region is ignored because those spacetimes violate the constraint in Equation (6). Note that the constraints reported in Ref. [59] are slightly different because that study used an earlier version of RELXILL_NK, and therefore the constraints reported here should be more reliable.

\subsection{Ark 564}

Ark 564 is another Narrow Line Seyfert 1 galaxy. The spectrum is dominated by the reflection component and, previous studies that assumed the Kerr metric, found that the inner edge of the accretion disk extents to very small radii. Ark 564 was observed by Suzaku on 26-28 June 2007 for about 80 ks. In Ref. [60], we analyzed this observation with RELXILL_NK. The data can be fitted with the following model

TBABS $\times($ RELXILL_NK + XILLVER $)$.

The constraints on the spin parameter $a_{*}$ and the Johannsen parameter $\alpha_{13}$ are shown in Figure 4 . The red, green, and blue lines indicate, respectively, the $68 \%, 90 \%$, and $99 \%$ confidence level curves for two relevant parameters. The grayed region is ignored because those spacetimes violate the constraint in Equation (6).

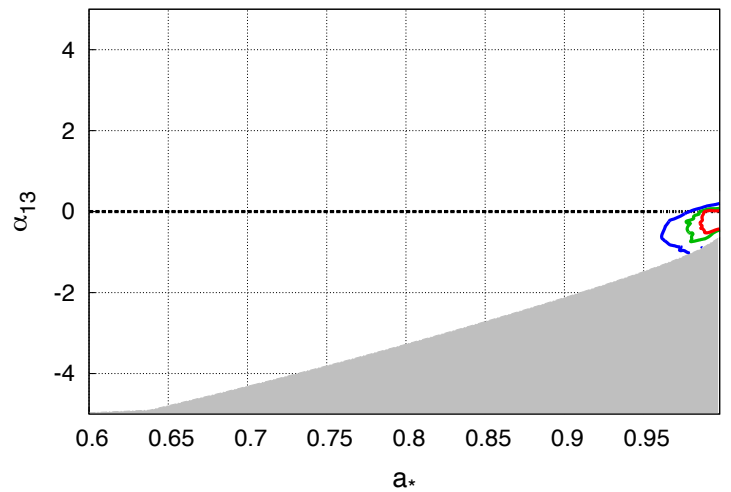

Figure 4. Constraints on the spin parameter $a_{*}$ and the Johannsen parameter $\alpha_{13}$ from the study in Ref. [60] of Suzaku data of Ark 564. The red, green, and blue lines indicate, respectively, the 68\%, 90\%, and $99 \%$ confidence level curves for two relevant parameters. The grayed region is ignored because those spacetimes violate the constraint in Equation (6). 


\subsection{GX 339-4}

GX 339-4 is an X-ray binary with a stellar-mass black hole and a stellar companion of mass $M_{\mathrm{c}}<2 M_{\odot}$ [63]. It is quite an active source and typically has an outburst every 2-3 years, so there are several observations in the archive. However, many observations are not suitable for our tests of the Kerr metric because the inner edge of the accretion disk is not at the innermost stable circular orbit but is truncated at some larger radius [64].

In Ref. [61], we analyzed a composite spectrum from the detector CPU-2 of RXTE with the highest observed luminosity in the hard state. We reached an unprecedented sensitivity of $\sim 0.1 \%$ and 40 million counts to capture the faint features in the reflection spectrum. We checked that the data are consistent with the inner edge being at the innermost stable circular orbit. The final model is

TBABS $\times$ GABS $\times($ RELXILL_NK + XILLVER $)$.

We performed Markov Chain Monte Carlo (MCMC) simulations and we obtained the following measurement for the black hole spin $a_{*}$ and the Johannsen parameter $\alpha_{13}$

$$
a_{*}=0.92_{-0.12}^{+0.07}, \quad \alpha_{13}=-0.76_{-0.60}^{+0.78}
$$

with a $90 \%$ confidence level for one relevant parameter. The 1-, 2-, and 3- $\sigma$ confidence level curves for two relevant parameters are shown in Figure 5.

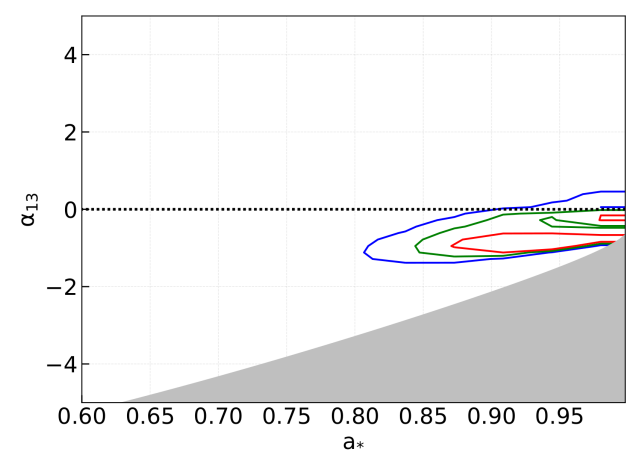

Figure 5. Constraints on the spin parameter $a_{*}$ and the Johannsen parameter $\alpha_{13}$ from the study in Ref. [61] of RXTE data of GX 339-4. The red, green, and blue lines indicate, respectively, the 1-, 2-, and 3- $\sigma$ confidence level curves for two relevant parameters after MCMC simulations. The grayed region is ignored because those spacetimes violate the constraint in Equation (6).

\subsection{GS $1354-645$}

GS 1354-645 is an X-ray binary with a dynamically confirmed black hole of mass $M \geq 7.6 \pm 0.7 M_{\odot}$ and a companion star of mass $M_{\mathrm{c}} \leq 1.2 M_{\odot}$ [65]. The source was discovered in its 1987 outburst by the Japanese X-ray mission Ginga [66]. In Ref. [62], we analyzed its July 11 NuSTAR observation of 30 ks. The data can be fitted with a simple reflection model

TBABS $\times$ RELXILL_NK.

The constraints on the spin parameter $a_{*}$ and the Johannsen parameter $\alpha_{13}$ are shown in Figure 6. For a quick comparison with the constraints from the other sources, the plot has the same spin and deformation parameter range as the other plots (the interested reader can find a more detailed plot of the constraints of this source in [62]). The constraint on the deformation parameter of this source is so strong here because the data suggest that the inner edge of the accretion disk is extremely close to the compact object. However, this result should be taken with some caution, as well as those from the other sources. We are only taking into account the statistical uncertainties while there are a number of simplifications in our model that can inevitably introduce systematic errors in the final 
measurement. For example, the Eddington scaled luminosity of this source in the observation analyzed is $L / L_{\text {Edd }} \leq 0.53$, which is consistent with the $L / L_{\text {Edd }}=0.05-0.20$ range of validity for the standard thin disk model, but it also allows for higher and lower luminosities where our disk model would not be adequate.

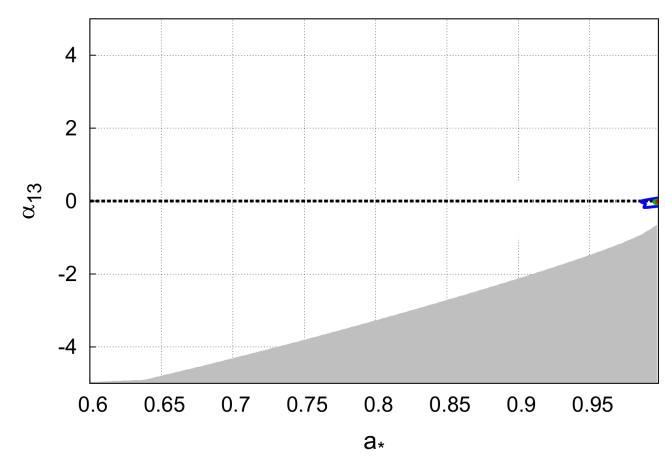

Figure 6. Constraints on the spin parameter $a_{*}$ and the Johannsen parameter $\alpha_{13}$ from the study in Ref. [62] of NuSTAR data of GS 1354-645. The red, green, and blue lines indicate, respectively, the $68 \%$, $90 \%$, and $99 \%$ confidence level curves for two relevant parameters. The grayed region is ignored because those spacetimes violate the constraint in Equation (6).

\section{Conclusions}

Einstein's theory of general relativity has been primarily tested in the weak gravitational regime, while the strong gravity regime is largely unexplored. However, there are many alternative theories of gravity that have the same predictions as Einstein's gravity in weak fields and present deviations when gravity becomes strong. The possibility of testing general relativity in the strong field regime is thus a major goal in modern physics.

Astrophysical black holes offer a quite unique possibility of testing strong gravitational fields. According to general relativity, the spacetime metric around these objects should be well approximated by the Kerr solution. In Ref. [57], we extended the relativistic reflection model RELXILL to test the Kerr black hole hypothesis from the study of the reflection spectrum of thin accretion disks. The new model is called RELXILL_NK. As of now, we have employed RELXILL_NK to analyze some X-ray data of the black holes in 1H0707-495, Ark 564, GX 339-4, and GS 1354-645. Current constraints on the spin parameter $a_{*}$ and the Johannsen deformation parameter $\alpha_{13}$ are summarized in Figures 2-6.

All our measurements are consistent with the hypothesis that the spacetime metric around astrophysical black holes is described by the Kerr solution. This is true even in the case of GS 1354-645, where the constraint on $\alpha_{13}$ is very strong (see Figure 6), and suggests that current uncertainties in the astrophysical model (e.g., infinitesimally thin disk, power-law or broken power-law for the emissivity profile, etc.) are not limiting our ability to constrain $\alpha_{13}$. However, the same conclusion may not hold for other deformation parameters that may be degenerate with the astrophysical model. The issues of the uncertainties of the astrophysical model and of their impact on the measurements of the model parameters are extremely important and not explored yet. Even in the case of spin measurements under the assumption of the Kerr metric, there are only a few preliminary studies to estimate the systematic errors and to construct more sophisticated models [67,68].

In the future, we plan to analyze the X-ray data of other sources, combine several observations of the same source to perform multi-epoch studies, improve our model RELXILL_NK in order to reduce the uncertainties of the model, and study a variety of deformations from the Kerr background. If we are able to construct a sufficiently sophisticated model to minimize the systematic effects, the next generation of X-ray mission may start a new era of precise tests of general relativity in the strong field regime with electromagnetic radiation. 
Author Contributions: C.B. proposed and supervised the project, and wrote the paper; A.B.A., D.A., and S.N. wrote and tested the model; Z.C., H.L., A.T., J.W.-J., and Y.X. analyzed the data and made the plots.

Funding: This research was funded by the National Natural Science Foundation of China (NSFC), Grant No. U1531117, and Fudan University, Grant No. IDH1512060. C.B. also acknowledges support from the Alexander von Humboldt Foundation. A.B.A. also acknowledges the support from the Shanghai Government Scholarship (SGS). S.N. acknowledges support from the Excellence Initiative at Eberhard-Karls Universität Tübingen. A.T. also acknowledges support from the China Scholarship Council (CSC), Grant No. 2016GXZR89.

Conflicts of Interest: The authors declare no conflict of interest.

\section{References}

1. Will, C.M. The Confrontation between General Relativity and Experiment. Living Rev. Relativ. 2014, 17, 4. [CrossRef] [PubMed]

2. Bambi, C. Testing black hole candidates with electromagnetic radiation. Rev. Mod. Phys. 2017, 89, 025001. [CrossRef]

3. Bambi, C.; Jiang, J.; Steiner, J.F. Testing the no-hair theorem with the continuum-fitting and the iron line methods: A short review. Class. Quant. Gravity 2016, 33, 064001. [CrossRef]

4. Yunes, N.; Siemens, X. Gravitational-Wave Tests of General Relativity with Ground-Based Detectors and Pulsar Timing-Arrays. Living Rev. Relativ. 2013, 16, 9. [CrossRef] [PubMed]

5. Yagi, K.; Stein, L.C. Black Hole Based Tests of General Relativity. Class. Quant. Gravity 2016, 33, 054001. [CrossRef]

6. Cardoso, V.; Gualtieri, L. Testing the black hole 'no-hair' hypothesis. Class. Quant. Gravity 2016, $33,174001$. [CrossRef]

7. Kerr, R.P. Gravitational field of a spinning mass as an example of algebraically special metrics. Phys. Rev. Lett. 1963, 11, 237-238. [CrossRef]

8. Carter, B. Axisymmetric Black Hole Has Only Two Degrees of Freedom. Phys. Rev. Lett. 1971, 26, 331-333. [CrossRef]

9. Robinson, D.C. Uniqueness of the Kerr black hole. Phys. Rev. Lett. 1975, 34, 905-906. [CrossRef]

10. Chrusciel, P.T.; Lopes Costa, J.; Heusler, M. Stationary Black Holes: Uniqueness and Beyond. Living Rev. Reativ. 2012, 15, 7. [CrossRef] [PubMed]

11. Volkov, M.S.; Gal'tsov, D.V. Gravitating non-Abelian solitons and black holes with Yang-Mills fields. Phys. Rep. 1999, 319, 1-83. [CrossRef]

12. Herdeiro, C.A.R.; Radu, E. Kerr black holes with scalar hair. Phys. Rev. Lett. 2014, 112, 221101. [CrossRef] [PubMed]

13. Price, R.H. Nonspherical perturbations of relativistic gravitational collapse. 1. Scalar and gravitational perturbations. Phys. Rev. D 1972, 5, 2419. [CrossRef]

14. Pretorius, F. Evolution of binary black hole spacetimes. Phys. Rev. Lett. 2005, 95, 121101. [CrossRef] [PubMed]

15. Bambi, C.; Dolgov, A.D.; Petrov, A.A. Black holes as antimatter factories. J. Cosmol. Astropart. Phys. 2009, 2009, 013. [CrossRef]

16. Bambi, C. Black Holes: A Laboratory for Testing Strong Gravity; Springer: Singapore, 2017.

17. Barausse, E.; Cardoso, V.; Pani, P. Can environmental effects spoil precision gravitational-wave astrophysics? Phys. Rev. D 2014, 89, 104059. [CrossRef]

18. Bambi, C.; Malafarina, D.; Tsukamoto, N. Note on the effect of a massive accretion disk in the measurements of black hole spins. Phys. Rev. D 2014, 89, 127302. [CrossRef]

19. Berti, E.; Barausse, E.; Cardoso, V.; Gualtieri, L.; Pani, L.; Sperhake, U.; Stein, L.C.; Wex, N.; Yagi, K.; Baker, T.; et al. Testing General Relativity with Present and Future Astrophysical Observations. Class. Quant. Gravity 2015, 32, 243001. [CrossRef]

20. Dvali, G.; Gomez, C. Black Hole's Quantum N-Portrait. Fortschr. Phys. 2013, 61, 742-767. [CrossRef]

21. Dvali, G.; Gomez, C. Black Hole's 1/N Hair. Phys. Lett. B 2013, 719, 419-423. [CrossRef]

22. Giddings, S.B. Possible observational windows for quantum effects from black holes. Phys. Rev. D 2014, 90, 124033. [CrossRef]

23. Herdeiro, C.A.R.; Radu, E. Asymptotically flat black holes with scalar hair: A review. Int. J. Mod. Phys. D 2015, 24, 1542014. [CrossRef] 
24. Remillard, R.A.; McClintock, J.E. X-ray Properties of Black-Hole Binaries. Ann. Rev. Astron. Astrophys. 2006, 44, 49-92. [CrossRef]

25. Kormendy, J.; Richstone, D. Inward bound: The Search for supermassive black holes in galactic nuclei. Ann. Rev. Astron. Astrophys. 1995, 33, 581. [CrossRef]

26. Rhoades, C.E., Jr.; Ruffini, R. Maximum mass of a neutron star. Phys. Rev. Lett. 1974, 32, 324-327. [CrossRef]

27. Lattimer, J.M. The nuclear equation of state and neutron star masses. Ann. Rev. Nucl. Part. Sci. 2012, 62, 485-515. [CrossRef]

28. Maoz, E. Dynamical constraints on alternatives to massive black holes in galactic nuclei. Astrophys. J. 1998, 494, L181-L184. [CrossRef]

29. Narayan, R.; McClintock, J.E. Advection-Dominated Accretion and the Black Hole Event Horizon. New Astron. Rev. 2008, 51, 733-751. [CrossRef]

30. Broderick, A.E.; Loeb, A.; Narayan, R. The Event Horizon of Sagittarius A*. Astrophys. J. 2009, 701, 1357-1366. [CrossRef]

31. Abbott, B.P.; et al. [LIGO Scientific and Virgo Collaborations]. Observation of Gravitational Waves from a Binary Black Hole Merger. Phys. Rev. Lett. 2016, 116, 061102. [CrossRef] [PubMed]

32. Abbott, B.P. et al. [LIGO Scientific and Virgo Collaborations]. Tests of general relativity with GW150914. Phys. Rev. Lett. 2016, 116, 221101. [CrossRef] [PubMed]

33. Cardoso, V.; Franzin, E.; Pani, P. Is the gravitational-wave ringdown a probe of the event horizon? Phys. Rev. Lett. 2016, 116, 171101. [CrossRef] [PubMed]

34. Yunes, N.; Yagi, K.; Pretorius, F. Theoretical Physics Implications of the Binary Black-Hole Mergers GW150914 and GW151226. Phys. Rev. D 2016, 94, 084002. [CrossRef]

35. Bambi, C. Astrophysical Black Holes: A Compact Pedagogical Review. Annalen Phys. 2018, 530, 1700430. [CrossRef]

36. Brenneman, L.W.; Reynolds, C.S. Constraining Black Hole Spin Via X-ray Spectroscopy. Astrophys. J. 2006, 652, 1028. [CrossRef]

37. Reynolds, C.S. Measuring Black Hole Spin using X-ray Reflection Spectroscopy. Space Sci. Rev. 2014, 183, 277-294. [CrossRef]

38. Schee, J.; Stuchlik, Z. Profiles of emission lines generated by rings orbiting braneworld Kerr black holes. Gen. Rel. Gravity 2009, 41, 1795-1818. [CrossRef]

39. Johannsen, T.; Psaltis, D. Testing the No-Hair Theorem with Observations in the Electromagnetic Spectrum. IV. Relativistically Broadened Iron Lines. Astrophys. J. 2013, 773, 57. [CrossRef]

40. Bambi, C. Testing the space-time geometry around black hole candidates with the analysis of the broad $\mathrm{K} \alpha$ iron line. Phys. Rev. D 2013, 87, 023007. [CrossRef]

41. Bambi, C.; Malafarina, D. K $\alpha$ iron line profile from accretion disks around regular and singular exotic compact objects. Phys. Rev. D 2013, 88, 064022. [CrossRef]

42. Jiang, J.; Bambi, C.; Steiner, J.F. Using iron line reverberation and spectroscopy to distinguish Kerr and non-Kerr black holes. J. Cosmol. Astropart. Phys. 2015, 2015, 025. [CrossRef]

43. Jiang, J.; Bambi, C.; Steiner, J.F. Testing the Kerr Nature of Black Hole Candidates using Iron Line Spectra in the CPR Framework. Astrophys. J. 2015, 811, 130. [CrossRef]

44. Zhou, M.; Cardenas-Avendano, A.; Bambi, C.; Kleihaus, B.; Kunz, J. Search for astrophysical rotating Ellis wormholes with X-ray reflection spectroscopy. Phys. Rev. D 2016, 94, 024036. [CrossRef]

45. Ni, Y.; Zhou, M.; Cardenas-Avendano, A.; Bambi, C.; Herdeiro, C.A.R.; Radu, E. Iron K $\alpha$ line of Kerr black holes with scalar hair. J. Cosmol. Astropart. Phys. 2016, 2016, 049. [CrossRef]

46. Cao, Z.; Cardenas-Avendano, A.; Zhou, M.; Bambi, C.; Herdeiro, C.A.R.; Radu, E. Iron K $\alpha$ line of boson stars. J. Cosmol. Astropart. Phys. 2016, 2016, 003. [CrossRef]

47. Ghasemi-Nodehi, M.; Bambi, C. Constraining the Kerr parameters via X-ray reflection spectroscopy. Phys. Rev. D 2016, 94, 104062. [CrossRef]

48. Shen, T.; Zhou, M.; Bambi, C.; Herdeiro, C.A.R.; Radu, E. Iron K $\alpha$ line of Proca stars. J. Cosmol. Astropart. Phys. 2017, 2017, 014. [CrossRef]

49. Zhou, M.; Bambi, C.; Herdeiro, C.A.R.; Radu, E. Iron K $\alpha$ line of Kerr black holes with Proca hair. Phys. Rev. D 2017, 95, 104035. [CrossRef]

50. Zhang, H.; Zhou, M.; Bambi, C.; Kleihaus, B.; Kunz, J.; Radu, E. Testing Einstein-dilaton-Gauss-Bonnet gravity with the reflection spectrum of accreting black holes. Phys. Rev. D 2017, 95, 104043. [CrossRef] 
51. Zhang, Y.; Zhou, M.; Bambi, C. Iron line spectroscopy of black holes in asymptotically safe gravity. Eur. Phys. J. C 2018, 78, 376. [CrossRef]

52. Dauser, T.; Garcia, J.; Wilms, J.; Bock, M.; Brenneman, L.W.; Falanga, M.; Fukumura, K.; Reynolds, C.S. Irradiation of an Accretion Disc by a Jet: General Properties and Implications for Spin Measurements of Black Holes. Mon. Not. R. Astron. Soc. 2013, 430, 1694. [CrossRef]

53. Garcia, J.; Dauser, T.; Lohfink, A.; Kallman, T.R.; Steiner, J.F.; McClintock, J.E.; Brenneman, L.; Wilms, J.; Eikmann, W.; Reynolds, C.S. Improved Reflection Models of Black-Hole Accretion Disks: Treating the Angular Distribution of X-rays. Astrophys. J. 2014, 782, 76. [CrossRef]

54. Garcia, J.; Kallman, T. X-ray reflected spectra from accretion disk models. I. Constant density atmospheres. Astrophys. J. 2010, 718, 695. [CrossRef]

55. Garcia, J.; Dauser, T.; Reynolds, C.S.; Kallman, T.R.; McClintock, J.E.; Wilms, J.; Eikmann, W. X-ray reflected spectra from accretion disk models. III. A complete grid of ionized reflection calculations. Astrophys. J. 2013, 768, 146. [CrossRef]

56. Dauser, T.; Wilms, J.; Reynolds, C.S.; Brenneman, L.W. Broad emission lines for negatively spinning black holes. Mon. Not. R. Astron. Soc. 2010, 409, 1534. [CrossRef]

57. Bambi, C.; Cardenas-Avendano, A.; Dauser, T.; Garcia, J.A.; Nampalliwar, S. Testing the Kerr black hole hypothesis using X-ray reflection spectroscopy. Astrophys. J. 2017, 842, 76. [CrossRef]

58. Johannsen, T. Regular Black Hole Metric with Three Constants of Motion. Phys. Rev. D 2013, 88, 044002. [CrossRef]

59. Cao, Z.; Nampalliwar, S.; Bambi, C.; Dauser, T.; Garcia, J.A. Testing general relativity with the reflection spectrum of the supermassive black hole in 1H0707-495. Phys. Rev. Lett. 2018, 120, 051101. [CrossRef] [PubMed]

60. Tripathi, A.; Nampalliwar, S.; Abdikamalov, A.B.; Ayzenberg, D.; Jiang, J.; Bambi, C. Testing the Kerr nature of the supermassive black hole in Ark 564. arXiv 2018, arXiv:1804.10380.

61. Wang-Ji, J.; Abdikamalov, A.B.; Ayzenberg, D.; Bambi, C.; Dauser, T.; Garcia, J.A.; Nampalliwar, S.; Steiner, J.F. Testing the Kerr metric using X-ray reflection spectroscopy: Spectral analysis of GX 339-4. arXiv 2018, arXiv:1806.00126.

62. Xu, Y.; Abdikamalov, A.B.; Ayzenberg, D.; Bambi, C.; Dauser, T.; Garcia, J.A.; Jiang, J.; Nampalliwar, S. A study of the strong gravity region of the black hole in GS 1354-645. 2018, in preparation.

63. Heida, M.; Jonker, P.G.; Torres, M.A.P.; Chiavassa, A. The Mass Function of GX 339-4 from Spectroscopic Observations of Its Donor Star. Astrophys. J. 2017, 846, 132. [CrossRef]

64. Wang-Ji, J.; García, J.A.; Steiner, J.F.; Tomsick, J.A.; Harrison, F.A.; Bambi, C.; Petrucci, P.-O.; Ferreira, J.; Clavel, M. The Evolution of GX 339-4 in the Low-hard State as Seen by NuSTAR and Swift. Astrophys. J. 2018, 855, 61. [CrossRef]

65. Casares, J.; Orosz, J.A.; Zurita, C.; Shahbaz, T.; Corral-Santana, J.M.; McClintock, J.E.; Garcia, M.R.; Martínez-Pais, I.G.; Charles, P.A.; Fender, R.P.; et al. Refined Orbital Solution and Quiescent Variability in the Black Hole Transient GS 1354-64 (= BW Cir). Astrophys. J. Suppl. 2009, 181, 238. [CrossRef]

66. Makino, F. 4U 0115+63 and X-Ray Nova. IAU Circular No. 4342, 1987; p. 1. Available online: http:/ /adsabs. harvard.edu/abs/1987IAUC.4342...1M (accessed on 10 July 2018).

67. Wu, S.M.; Wang, T.G. Iron line profiles and self-shadowing from relativistic thick accretion discs. Mon. Not. R. Astron. Soc. 2007, 378, 841-851. [CrossRef]

68. Taylor, C.; Reynolds, C.S. Exploring The Effects Of Disk Thickness On The Black Hole Reflection Spectrum. Astrophys. J. 2018, 855, 120. [CrossRef]

(C) 2018 by the authors. Licensee MDPI, Basel, Switzerland. This article is an open access article distributed under the terms and conditions of the Creative Commons Attribution (CC BY) license (http://creativecommons.org/licenses/by/4.0/). 